\title{
Orbit classification of qutrit via the Gram matrix.
}

\begin{abstract}
We classify the orbits generated by unitary transformation on the density matrices of the three-state quantum systems (qutrits) via the Gram matrix. The Gram matrix is a real symmetric matrix formed from the Hilbert \{ Schmidt scalar products of the vectors lying in the tangent space to the orbits. The rank of the Gram matrix determines the dimensions of the orbits, which fall into three classes for qutrits.
\end{abstract}

Keyword: Qutrit; Gram matrix; Density matrix. 